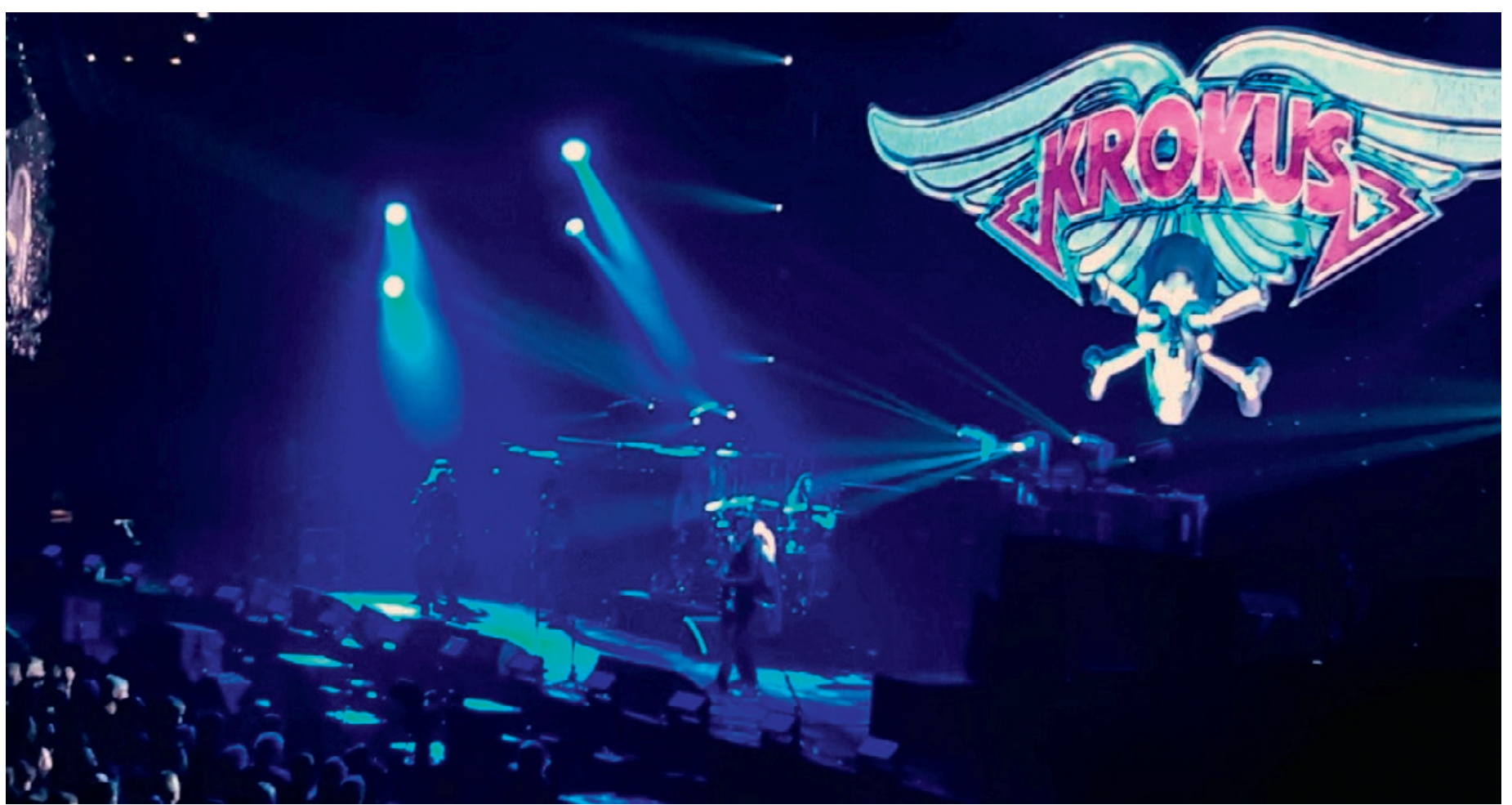

\title{
K wie Krokus oder der geriatric rock
}

\section{Dominik Heim}

PD Dr. med., Lex latrik, Versicherungsmedizin, Luzern

Randy Newman [1], der oscargekrönte, amerikanische Songwriter, sagte zu seinen Zuhörern beim Konzert mit dem London Symphony Orchestra 2008: «You must have noticed as I have, that nobody is retiring from Rock'n'Roll.» Momol! Krokus, die bekannteste Hardrockband der Schweiz, international berühmt seit ihrer Amerika-Tour 1980, haben dies Anfang Dezember im Zürcher Hallenstadion gemacht. Und es war die wohl lauteste Pensionierung, die es je gegeben hat! Bombastisch, mit viel Feuerwerk, Rauch und glitzernden Konfettischnitzeln, ohrenbetäubend trotz Ohrenstöpseln, spontan mit zweimal vier Zugaben anstatt dem üblichen encore und mit einer never ending standing ovation, Krokus sind abgetreten. Schade.

Aber Randy Newman hat auch recht! Das tun nicht alle Rockgrössen: Andere spielen jetzt ihre Alterswerke ein. Soeben haben die beiden übriggebliebenen Mitglieder der Who, Roger Daltrey und Pete Townshend, ihren letzten Wurf mit dem simplen Titel «WHO» veröffentlicht. 1965 haben sie mit «my generation» die Rockwelt erschüttert. Es sei das beste Album seit dem Tod ihres Schlagzeugers Keith Moon 1978, schreiben die einen; sie hätten es besser sein lassen, schreiben die anderen.
Alterswerke werden halt meist kritisch aufgenommen: Sind sie so gut wie früher, als die Bandmitglieder noch lange Haare hatten, Gitarren auf der Bühne zertrümmerten - diesen legendären Zerstörungsakt nannte man dann "autodestruktive Kunst»-, oder ist es einfach ein Abklatsch der früheren, heroischen Zeiten? Diese Gefahr des Alters-Abklatschs beschreibt das Lied von Randy Newman sehr zutreffend: «I'm dead, but I don't know it.» Aber nein, (meist) sind sie gar nicht tot - zuweilen sehen sie aber fast so aus. Schauen Sie sich Iggy Pop an, den man auch als godfather of punk apostrophiert. In seinen tiefen Gesichtsfurchen lässt sich sein bewegtes Leben als Sänger, Gitarrist, Schlagzeuger und Komponist förmlich ablesen. Meist ist diese Altersmusik aber in der Tat anders, ruhiger, auch abgeklärter, wohl darum heisst Iggys Album auch «free». At last, möchte man beifügen, wenn er am Anfang mit sphärischer Untermalung sagt: «I wanna be free free.» Das renommierte Musikmagazin «Rolling Stone» charakterisiert diesen neuen free-Stil: «Iggy zittert, rezitiert, kontempliert, schreit, der Jazztrompeter Leron Thomas spielt mal gedämpfte, mal klar gerichtete Soli. Cool. Auch wenn (und weil) es wenig rockt.» 


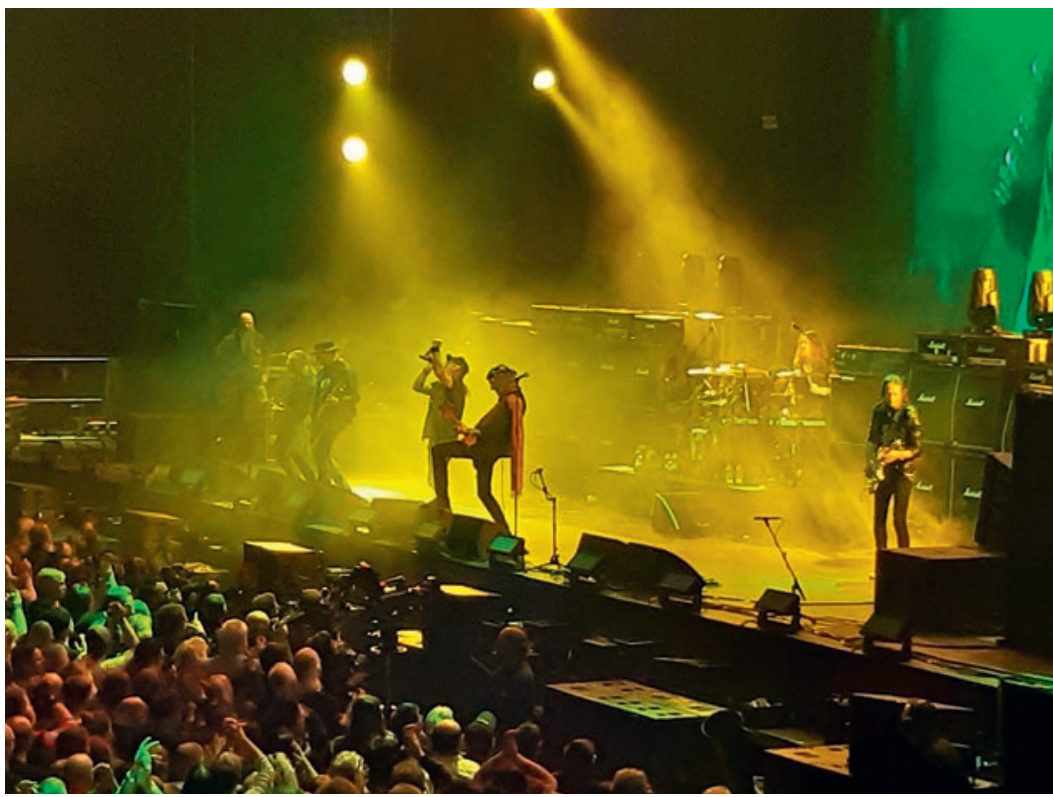

Krokuskonzert vom 7. Dezember 2019 im Hallenstadion Zürich (๔ D. Heim).

Ja, Alterswerke sind anders, alles andere wäre auch etwas unglaubwürdig, ausser bei Mick Jagger. Der rennt mit seiner neuen Aortenklappe, in der TAVI-Technik implantiert, wild gestikulierend über die Bühne wie beim denkwürdigen Konzert der Rolling Stones im Letzigrund vor zwei Jahren.

All diesen Werken des geriatric rock - dieser Ausdruck stammt übrigens auch von Randy Newman - ist etwas gemeinsam. George Harrison von den Beatles sang in weiser Voraussicht, wohl aber in einer anderen Absicht: "All things must pass. None of the life's strings can last.»

Das ist es, was wohl Roger Daltrey meint, wenn er auf "WHO" singt: "All this music will fade, just like the edge of a blade.» Irgendwann ist dann fertig. Und bei dieser stumpfen Klinge erinnern wir Chirurgen uns daran, dass auch bei uns das Ende der manuellen Tätigkeit irgendwann unweigerlich kommt. Zum Zeitpunkt haben die Rolling Stones einen ganz praktischen Vorschlag: "I was a surgeon, till I start to shake», singt Mick Jagger in "You got me rocking» (1994). Und wenn sich dann bei diesem Zeitpunkt des «Das Messer aus der Hand legen» eine gewisse Melancholie und Unruhe ausbreiten sollten, dann gibt es ja immer noch viele Möglichkeiten, diesen in den vielen Jahren angesammelten Erfahrungsschatz in der einen oder anderen Form anzuwenden und weiterzugeben an die nächste oder übernächste Generation. Besagte Who sangen zwar vor vielen Jahrzehnten in "my generation»: "Yeah, I hope I die before I get old.» Das trat ganz offensichtlich nicht ein und muss es auch nicht. Iggy Pop schreibt fast entschuldigend zu seinem Verbleiben in der Musikszene $\mathrm{zu}$ "free»: "This is an album in which other artists speak for me, but I lend my voice.» Art und Zeitpunkt der chirurgischen und musikalischen Pensionierung sind demnach ein gänzlich individueller Entscheid, ganz so, wie es diese "geriatrischen" Rock-Giganten tun und taten. Leonard Cohen (19342016), er verzeihe das Attribut Rock, singt in seinem posthum erschienenen Album voller Demut «thanks for the dance». Erfreulich war dieses Ende aber sicher nicht, wenn man in seinen melancholischen, mehr gesprochenen als gesungenen Liedern hört: «I'm living on pills» und «I can't leave my house or can't answer the phone», um dann mit "the neighbor returns my smile of defeat» zu resignieren. Nein, nicht schön. Aber vielleicht wollen wir auch gar nicht an diese Vergänglichkeit erinnert werden, an den hohen Blutdruck, ans Cholesterin und an die Prostata. Vielleicht haben die Who recht, wenn sie singen: «I don't care, I know you're gonna hate this song.» Denn es könnte in der Tat das letzte Album sein. Freuen würden wir uns zwar auf mehr. So, wie wir es tun, wenn wir «the later years» (2019) von den Pink Floyd auflegen. Diese Stücke können wir längst mitsingen. Aber vielleicht gilt dieser Satz auch gar nicht uns, den Hörern, sondern Pete Townshend, der in "all this music must fade» etwas Rap haben wollte, was bei Roger Daltrey aber gar nicht gut ankam: «Well, no fucking way I'm going to rap. No way. Let the youngsters wear those clothes.» Es ist in diesem Zusammenhang auch interessant zu wissen, dass sie «WHO» nicht zusammen im Studio aufgenommen haben, sondern jeder separat für sich. I don't care - tönen tut es trotzdem einfach gut.

Das sind Gedanken frühmorgens im Zug auf dem Weg zur Arbeit, draussen ist jetzt auch im Flachland der erste Schnee angekommen, man macht das Schreiblicht oben beim SBB-Sitz an, es ist stimmig wie das stimmige Gutachten [2], das vor mir liegt, ruhig und free. Und dazu trägt eben auch die vertraute Stimme Daltreys und die jetzt eher ruhige Musik von the Who bei, die rückblickend singen: «This life was like climbing, so glad I put my time in [...]» Ja, me too.

(PS. Man höre die Musik laut, wie damals, als wir «my generation» in den Ohren hatten!)

\section{Literatur}

1 Heim D. R wie Randy Newman oder Musik aus Hollywood im Ops. Schweiz Ärzteztg. 2017;98(41):1350

2 Heim D. V wie Versicherungsmedizin oder das stimmige Bild. Schweiz Ärzteztg. 2018;99(43):1515-6.

Restliche Literatur (Liedertexte, Rezensionen) beim Verfasser resp. in den booklets der CDs. 